\title{
DETERMINATION OF A CONTROL PARAMETER IN A PARABOLIC PARTIAL DIFFERENTIAL EQUATION
}

\author{
J. R. CANNON ${ }^{1}$, YANPING LIN $^{2}$ and SHINGMIN WANG ${ }^{3}$
}

(Received 25 November 1988; revised 22 June 1989)

\begin{abstract}
The authors consider in this paper the inverse problem of finding a pair of functions $(u, p)$ such that

$$
\begin{gathered}
u_{t}=u_{x x}+p u+F(x, t), \quad 0<x<1, \quad 0<t \leq T, \\
u(x, 0)=f(x), \quad 0 \leq x \leq 1, \\
\alpha_{1}(t) u_{x}(0, t)+\beta_{1}(t) u(0, t)+\gamma_{1}(t) u(1, t)=g_{1}(t), \quad 0<t \leq T, \\
\alpha_{2}(t) u_{x}(1, t)+\beta_{2}(t) u(0, t)+\gamma_{2}(t) u(1, t)=g_{2}(t), \quad 0<t \leq T, \\
\int_{0}^{s(t)} u(x, t) d x=E(t), \quad 0 \leq t \leq T, \quad 0<s(t) \leq 1 .
\end{gathered}
$$
\end{abstract}

where $F, f, E, s, \alpha_{i}, \beta_{i}, \gamma_{i}, g_{i}, i=1,2$, are given functions.

The existence and uniqueness of a smooth global solution pair $(u, p)$ which depends continuously upon the data are demonstrated under certain assumptions on the data.

\section{Introduction}

This paper is concerned with the following inverse problem of finding a pair $(u, p)$ such that

\footnotetext{
'Department of Mathematics, Lamar University, Beaumont, TX 77710.

${ }^{2}$ Department of Applied Mathematics, University of Waterloo, Waterloo, Canada N2L 3G1.

${ }^{3}$ Division of Mathematics and Computer Science, Northeast Missouri State University, Kirksville, MO 63501.

(C) Copyright Australian Mathematical Society 1991, Serial-fee code 0334-2700/91
} 


$$
\begin{gathered}
u_{t}=u_{x x}+p(t) u+F(x, t), \quad 0<x<1, \quad 0<t \leq T, \\
u(x, 0)=f(x), \quad 0 \leq x \leq 1, \\
\alpha_{1}(t) u_{x}(0, t)+\beta_{1}(t) u(0, t)+\gamma_{1}(t) u(1, t)=g_{1}(t), \quad 0<t \leq T, \\
\alpha_{2}(t) u_{x}(1, t)+\beta_{2}(t) u(0, t)+\gamma_{2}(t) u(1, t)=g_{2}(t), \quad 0<t \leq T, \\
\int_{0}^{s(t)} u d x=E(t), \quad 0<t \leq T, 0<s(t) \leq 1,
\end{gathered}
$$

where $F, f, s, E, g_{i}, \alpha_{i}, \beta_{i}, \gamma_{i}, i=1,2$, are given functions.

Nonlocal boundary specifications like (1.5) arise from many important applications in heat transfer, thermoelasticity, control theory, life science, etc. For example, in a heat transfer process, if we let $u$ represent the temperature distribution, then (1.1)-(1.5) can be regarded as a control problem with source control. A source control parameter $p(t)$ needs to be determined so that a desired thermal energy can be obtained for a portion of the spatial domain. In an environment where heat transfer takes place between liquids and solids, the heat flux is often taken to be proportional to the difference in the boundary temperature of the solid and the temperature of the liquid, and here, $\alpha_{i}, \beta_{i}, \gamma_{i}, i=1,2$, represent those proportionality factors. For more examples, the reader is referred to [2]-[6], [10]-[12] and [14]-[16].

The authors of [20], [21], and [22] investigated problems similar to (1.1)(1.5) by employing abstract semi-group theory. In those problems, the equation similar to (1.1) was almost linear, and the coefficients were independent of time. Cannon and Lin extended the investigation to quasilinear parabolic equations [7], [8]. However, because of the restriction of the method they used there, only local solutions were obtained. In this paper, we will take a new approach to demonstrate the existence of the global solution of (1.1)-(1.5).

We begin our investigation with a pair of transformations:

$$
r(t)=\exp \left\{-\int_{0}^{t} p(\tau) d \tau\right\}
$$

and

$$
v(x, t)=u(x, t) r(t)
$$


Introducing (1.6) and (1.7) into (1.1)-(1.5), we obtain

$$
\begin{gathered}
v_{t}=v_{x x}+r(t) F(x, t), \quad 0<x<1, \quad 0<t \leq T, \\
v(x, 0)=f(x), \quad 0 \leq x \leq 1, \\
\alpha_{1}(t) v_{x}(0, t)+\beta_{1}(t) v(0, t)+\gamma_{1}(t) v(1, t)=r(t) g_{1}(t), \quad 0<t \leq T, \\
\alpha_{2}(t) v_{x}(1, t)+\beta_{2}(t) v(0, t)+\gamma_{2}(t) v(1, t)=r(t) g_{2}(t), \quad 0<t \leq T, \\
r(t)=[E(t)]^{-1} \int_{0}^{s(t)} v d x, \quad 0<t \leq T, \quad 0<s(t) \leq 1 .
\end{gathered}
$$

Equations (1.8)-(1.11) have been studied substantially by many authors, e.g., in [18], [19] and [23]. Instead of re-doing any of their work, this paper is devoted to the solution of the inverse problem that involves an over-specified condition (1.5), which now is represented by (1.12). The inclusion of (1.5) has created a new scenario, and thus requires special consideration on its solution. It is this consideration that constitutes our focus in this paper. In what follows, we shall show that under some reasonable assumptions there exists a unique solution pair $(v, r)$ to $(1.8)-(1.12)$ such that $r(t) \in C^{1}[0,1]$ and $r(t)>0$. Thus a unique solution pair $(u, p)$ can be obtained through the following inverse transformations to (1.6) and (1.7):

$$
\begin{gathered}
u(x, t)=\frac{v(x, t)}{r(t)}, \\
p(t)=-\frac{r^{\prime}(t)}{r(t)} .
\end{gathered}
$$

Now we proceed to approximate the solution pair $(v, r)$ by the following sequence of iterations:

$$
r^{(0)} \equiv 1
$$

For $n \geq 1, v^{(n)}$ and $r^{(n)}$ are defined by the equations

$$
\begin{array}{rr}
v_{t}^{(n)}=v_{x x}^{(n)}+r^{(n-1)} F, \quad 0<x<1, \quad 0<t \leq T, & (1.16) \\
v^{(n)}(x, 0)=f(x), \quad 0 \leq x \leq 1, & (1.17) \\
\alpha_{1}(t) v_{x}^{(n)}(0, t)+\beta_{1}(t) v^{(n)}(0, t)+\gamma_{1}(t) v^{(n)}(1, t)=r^{(n-1)} g_{1}(t), & 0<t \leq T, \\
\alpha_{2}(t) v_{x}^{(n)}(1, t)+\beta_{2}(t) v^{(n)}(0, t)+\gamma_{2}(t) v^{(n)}(1, t)=r^{(n-1)} g_{2}(t), & 0<t \leq T,
\end{array}
$$

and

$$
r^{(n)}=[E(t)]^{-1} \int_{0}^{s(t)} v^{(n)}(x, t) d x, \quad 0<t \leq T, 0<s(t) \leq 1 .
$$


For convenience, in what follows we shall use the following definitions:

$$
\begin{array}{ll}
K(x, t)=\frac{1}{\sqrt{4 \pi t}} \exp \left\{-\frac{x^{2}}{4 t}\right\}, & t>0, \\
\theta(x, t)=\sum_{m=-\infty}^{\infty} K(x+2 m, t), & t>0,
\end{array}
$$

for $\psi(x)$ defined on $[0,1], \psi(t)$ defined on $[0, T], \psi(x, t)$ defined on $[0,1] \times[0, T]$,

$$
\begin{gathered}
\|\psi(x)\|=\max _{x \in[0,1]}\{|\psi(x)|\}, \\
\|\psi(t)\|=\max _{t \in[0, T]}\{|\psi(t)|\}, \\
\|\psi(x, t)\|=\max _{(x, t) \in[0,1] \times[0, T]}\{|\psi(x, t)|\},
\end{gathered}
$$

and for a vector $\left(\psi_{1}(t), \psi_{2}(t)\right), t \in[0, T]$,

$$
\|\psi(t)\|=\max \left\{\left\|\psi_{1}(t)\right\|,\left\|\psi_{2}(t)\right\|\right\} .
$$

Assumption (A1). We assume that $F \in C^{a, a / 2}([0,1] \times[0, T])$, for $0<$ $a \leq 1, f \in C^{1}[0,1], g_{1}, g_{2} \in C[0, T], E(0)=\int_{0}^{s(0)} f(x) d x>0, E(t)>$ $0, s(t), E(t) \in C^{1}[0, T], \alpha_{i}, \beta_{i}, \gamma_{i} \in C[0, T], i=1,2$, and

$$
\begin{aligned}
& \alpha_{1}(0) f_{x}(0)+\beta_{1}(0) f(0)+\gamma_{1}(0) f(1)=g_{1}(0), \\
& \alpha_{2}(0) f_{x}(1)+\beta_{2}(0) f(0)+\gamma_{2}(0) f(1)=g_{2}(0) .
\end{aligned}
$$

We end this introduction with the statement of our first result which will be demonstrated in Section 2 and Section 3.

THEOREM 1. Under assumption (A1), if $\alpha_{1}(t) \neq 0, \alpha_{2}(t) \neq 0$, or $\alpha_{1}(t)=$ $\alpha_{2}(t)=0$ and $\beta_{1} \gamma_{2} \neq \beta_{2} \gamma_{1}$, then there exists a unique solution pair $(v, r)$ to $(1.8)-(1.12)$ in $[0,1] \times[0, \varepsilon]$ such that $r(t) \in C^{1}[0, \varepsilon]$ for some positive constant $\varepsilon$.

REMARK. This local solution will be extended to a global one.

\section{The mixed boundary value case}

\subsection{Existence and uniqueness}

We shall demonstrate Theorem 1 for the case of $\alpha_{1}(t) \neq 0$ and $\alpha_{2}(t) \neq 0$. Without loss of generality, we can assume that $\alpha_{1}(t)=\alpha_{2}(t)=1$. 
Proof of Theorem 1. Under the assumption (A1), it follows from [1] that the unique bounded solution of $(1.16)-(1.19)$ has the form

$$
\begin{aligned}
v^{(n)}(x, t)=w(x, t) & -2 \int_{0}^{t} \theta(x, t-r) \phi_{1}^{(n)}(\tau) d \tau \\
& +2 \int_{0}^{t} \theta(x-1, t-\tau) \phi_{2}^{(n)}(\tau) d \tau+z^{(n)}(x, t),
\end{aligned}
$$

where

$$
\begin{gathered}
w(x, t)=\int_{0}^{1}\{\theta(x-\xi, t)+\theta(x+\xi, t)\} f(\xi) d \xi, \\
z^{(n)}(x, t)=\int_{0}^{t} \int_{0}^{1}\{\theta(x-\xi, t-\tau)+\theta(x+\xi, t-\tau)\} r^{(n-1)}(\tau) F(\xi, \tau) d \xi d \tau,
\end{gathered}
$$

and $\phi_{1}^{(n)}(t), \phi_{2}^{(n)}(t)$ are the solutions of the following system of Volterra integral equations of the second kind:

$$
\begin{aligned}
& r^{(n-1)}(t) g_{i}(t)=\phi_{i}^{(n)}(t)+ \beta_{i}(t)\left[w(0, t)-2 \int_{0}^{t} \theta(0, t-\tau) \phi_{1}^{(n)}(\tau) d \tau\right. \\
&\left.+2 \int_{0}^{t} \theta(-1, t-\tau) \phi_{2}^{(n)}(\tau) d \tau+z^{(n)}(0, t)\right] \\
&+\gamma_{i}(t)\left[w(1, t)-2 \int_{0}^{t} \theta(1, t-\tau) \phi_{1}^{(n)}(\tau) d \tau\right. \\
&\left.+2 \int_{0}^{t} \theta(0, t-\tau) \phi_{2}^{(n)}(\tau) d \tau+z^{(n)}(1, t)\right] \\
& i=1,2 .
\end{aligned}
$$

Following [1], using

$$
\exp \{-\xi\} \leq p ! \xi^{-p}, \quad \text { for } \xi>0, p=0,1,2, \ldots,
$$

and

$$
\theta(x, t)=1+2 \sum_{k=1}^{\infty} \exp \left\{-k^{2} \pi^{2} t\right\} \cos k \pi x,
$$

we can easily derive the following:

$$
|\theta(x, t-\tau)| \leq \frac{C}{2 \sqrt{t-\tau}}, \quad t>\tau,
$$

where $C$ is a positive constant,

$$
\begin{gathered}
\|w(x, t)\| \leq 2\|f\|, \\
\int_{0}^{t}[\theta(0, t-\tau)+\theta(1, t-\tau)] d \tau \leq 2 C \sqrt{t}, \\
\left\|z^{(n+1)}-z^{(n)}\right\| \leq 2 C \sqrt{t}\left\|r^{(n)}-r^{(n-1)}\right\| .\|F\| .
\end{gathered}
$$


From (2.4) we obtain

$$
\begin{aligned}
& \left|\phi_{i}^{(n+1)}-\phi_{i}^{(n)}\right| \leq\left|r^{(n)}-r^{(n-1)}\right|\left|g_{i}\right| \\
& \quad+2\left\|\phi_{1}^{(n+1)}-\phi_{1}^{(n)}\right\|\left(\left|\beta_{i}\right| \int_{0}^{t} \theta(0, t-\tau) d \tau+\left|\gamma_{i}\right| \int_{0}^{t} \theta(1, t-\tau) d \tau\right) \\
& \quad+2\left\|\phi_{2}^{(n+1)}-\phi_{2}^{(n)}\right\|\left(\left|\beta_{i}\right| \int_{0}^{t} \theta(-1, t-\tau) d \tau+\left|\gamma_{i}\right| \int_{0}^{t} \theta(0, t-\tau) d \tau\right) \\
& \quad+\left|z^{(n+1)}(0, t)-z^{(n)}(0, t)\right| \\
& \quad+\left|z^{(n+1)}(1, t)-z^{(n)}(1, t)\right|, \quad i=1,2 .
\end{aligned}
$$

Applying (2.8), (2.9), and (2.10) to (2.11), we obtain

$$
\begin{aligned}
\left\|\phi^{(n+1)}-\phi^{(n)}\right\| \leq & 4 C \sqrt{t}(\|\beta\|+\|\gamma\|)\left\|\phi^{(n+1)}-\phi^{(n)}\right\| \\
& +(4 C \sqrt{t}\|F\|+\|g\|)\left\|r^{(n)}-r^{(n-1)}\right\|,
\end{aligned}
$$

and this yields

$$
\left\|\phi^{(n+1)}-\phi^{(n)}\right\|<C_{1}\left\|r^{(n)}-r^{(n-1)}\right\|
$$

where

$$
\begin{gathered}
C_{1}=\frac{4 C \sqrt{t}\|F\|+\|g\|}{1-4 C \sqrt{t}(\|\beta\|+\|\gamma\|)}, \\
4 C \sqrt{t}(\|\beta\|+\|\gamma\|)<1, \quad \text { for } t \leq \varepsilon,
\end{gathered}
$$

and $\varepsilon$ is a positive constant which is sufficiently small.

REMARK. In what follows, a finite number of different $\varepsilon$ 's will be needed. To prevent the accumulation of symbols, we shall assume that this very first $\varepsilon$ will incorporate the minimum over all those $\varepsilon$ 's in order to validate all the inequalities concerned.

Defining

$$
\begin{aligned}
y^{(n)}=v^{(n+1)}-v^{(n)}, & & n=1,2, \ldots, \\
q^{(n)}=r^{(n+1)}-r^{(n)}, & & n=0,1, \ldots,
\end{aligned}
$$

we obtain from (2.1) and (1.20)

$$
\begin{aligned}
y^{(n)}= & 2 \int_{0}^{t} \theta(x-1, t-\tau)\left[\phi_{2}^{(n+1)}-\phi_{2}^{(n)}\right] d \tau \\
- & 2 \int_{0}^{t} \theta(x, t-\tau)\left[\phi_{1}^{(n+1)}-\phi_{1}^{(n)}\right] d \tau \\
+ & \int_{0}^{t} \int_{0}^{1}\{\theta(x-\xi, t-\tau)+\theta(x+\xi, t-\tau)\} \\
& \times\left[r^{(n)}-r^{(n-1)}\right] F(\xi, \tau) d \xi d \tau,
\end{aligned}
$$




$$
q^{(n)}=[E(t)]^{-1} \int_{0}^{s(t)} y^{(n)}(x, t) d x .
$$

It follows from (2.6), (2.13), (2.16) and (2.17) that

$$
\left\|q^{(n)}\right\| \leq 2 C \sqrt{t}\left\|[E(t)]^{-1}\right\|\left(2 C_{1}+\|F\|\right)\left\|q^{(n-1)}\right\| .
$$

It can be shown by elementary estimates that there exists a constant $\varepsilon>0$ such that for $t \leq \varepsilon$

$$
\begin{aligned}
& 2 C \sqrt{t}\left\|[E(t)]^{-1}\right\|\left(2 C_{1}+\|F\|\right) \\
& \quad=2\left\|[E(t)]^{-1}\right\| C \sqrt{t}\left[\frac{8 C \sqrt{t}\|F\|+2\|g\|}{1-4 C \sqrt{t}(\|\beta\|+\|\gamma\|)}+\|F\|\right] \leq \frac{1}{2},
\end{aligned}
$$

and thus

$$
\left\|q^{(n)}\right\| \leq \frac{1}{2}\left\|q^{(n-1)}\right\| \leq \frac{1}{2^{2}}\left\|q^{(n-2)}\right\| \leq \cdots \leq \frac{1}{2^{n}}\left\|q^{(0)}\right\| .
$$

This implies that $\left\{r^{(n)}\right\}$ is a Cauchy sequence in $C[0, \varepsilon]$, and hence we can conclude that

$\exists r(t) \in C[0, \varepsilon], \quad$ such that $r^{(n)}(t) \rightarrow r(t)$ uniformly.

It follows immediately from (2.10) and (2.13) that

$$
\exists z(x, t) \in C([0,1] \times[0, \varepsilon]), \quad z^{(n)}(x, t) \rightarrow z(x, t)
$$

uniformly, and

$$
\exists \phi_{i}(t) \in C[0, \varepsilon], \quad \phi_{i}^{(n)}(t) \rightarrow \phi(t) \text { uniformly, } \quad i=1,2 .
$$

Summarising this, we see from (2.1) that

$$
\exists v(x, t) \in C([0,1] \times[0, \varepsilon]), \quad v^{(n)}(x, t) \rightarrow v(x, t)
$$

uniformly.

Differentiating (2.1) with respect to $x$, we obtain

$$
\begin{aligned}
v_{x}^{(n)}= & w_{x}-2 \int_{0}^{t} \frac{\partial \theta}{\partial x}(x, t-\tau) \phi_{1}^{(n)}(\tau) d \tau \\
& +2 \int_{0}^{t} \frac{\partial \theta}{\partial x}(x-1, t-\tau) \phi_{2}^{(n)}(\tau) d \tau+z_{x}^{(n)}(x, t) .
\end{aligned}
$$

It is easy to show from [1] that there exists a constant $C_{2}>0$ such that

$$
\begin{gathered}
\int_{0}^{t} \frac{\partial \theta}{\partial x}(x, t-\tau) d \tau \leq C_{2}, \\
\int_{0}^{t} \frac{\partial \theta}{\partial x}(x-1, t-\tau) d \tau \leq C_{2},
\end{gathered}
$$


and

$$
\int_{0}^{t} \int_{0}^{1}\{\theta(x-\xi, t-\tau)+\theta(x+\xi, t-\tau)\} d \xi d \tau \leq C_{2} .
$$

Because of this, the uniform convergence of $r^{(n)}, \phi_{i}^{(n)}, i=1,2$, and (2.3), we can see that

$$
\begin{aligned}
v_{x}^{(n)} \rightarrow v_{x}(x, t)= & w_{x}-2 \int_{0}^{t} \frac{\partial \theta}{\partial x}(x, t-\tau) \phi_{1}(\tau) d \tau \\
& +2 \int_{0}^{t} \frac{\partial \theta}{\partial x}(x-1, t-\tau) \phi_{2}(\tau) d \tau+z_{x}(x, t),
\end{aligned}
$$

and that $v$ satisfies (1.8)-(1.11). As to the condition (1.12), we have that for $\delta>0$ and sufficiently small,

$$
\begin{aligned}
\int_{\delta}^{s(t)} v_{t} d x & =\int_{\delta}^{s(t)} v_{x x} d x+\int_{\delta}^{s(t)} r(t) F(x, t) d x \\
& =v_{x}(s(t), t)-v_{x}(\delta, t)+\int_{\delta}^{s(t)} r(t) F(x, t) d x .
\end{aligned}
$$

Letting $\delta \rightarrow 0$, we obtain

$$
\int_{0}^{s(t)} v_{t} d x=\left[v_{x}(s(t), t)-v_{x}(0, t)+\int_{0}^{s(t)} r(t) F(x, t) d x\right] \in C[0, \varepsilon]
$$

which implies that $r(t)=[E(t)]^{-1} \int_{0}^{s(t)} v d x$ is differentiable with respect to $t$, and $r^{\prime}(t) \in C[0, \varepsilon]$.

Now we show that the solution pair $(v, r)$ so obtained for (1.8)-(1.12) is unique. Suppose that $(\mu, \rho)$ is also a solution pair of $(1.8)-(1.12)$, and that $\mu_{x}(0, t)=\psi_{1}(t), \mu_{x}(1, t)=\psi_{2}(t)$. Then by the uniqueness of the representation of the solution, we have

$$
\begin{gathered}
v-\mu=2 \int_{0}^{t} \theta(x-1, t-\tau)\left(\phi_{2}-\psi_{2}\right) d \tau-2 \int_{0}^{t} \theta(x, t-\tau)\left(\phi_{1}-\psi_{1}\right) d \tau \\
+\int_{0}^{t} \int_{0}^{1}\{\theta(x-\xi, t-\tau)+\theta(x+\xi, t-\tau)\}[r-\rho] F(\xi, \tau) d \xi d \tau \\
r-\rho=[E(t)]^{-1} \int_{0}^{t}(v-\mu) d x
\end{gathered}
$$

Following the same procedure leading to $(2.20)$, we obtain

$$
\|r-\rho\| \leq \frac{1}{2}\|r-\rho\|,
$$

which implies that $r=\rho$. A similar argument establishes the equality $v=\mu$. Theorem 1 now has been proved for this case. 
In order to employ the inverse transformations (1.13) and (1.14), $r(t)$ is required to be positive in $[0, \varepsilon]$. The positivity of $r(t)$ depends on the data given to the original problem. It would be too lengthy to list all the possible combinations of the data. Instead, we shall investigate the following situation, though it will not be difficult to establish sufficient conditions for other specified settings.

First, we notice that $r(t)$, being the limit of $\left\{r^{(n)}(t)\right\}$, is bounded, i.e.,

$$
\exists\|r\|>0 \text { such that }|r(t)| \leq\|r\| \text { in }[0, \varepsilon] .
$$

Letting

$$
\tilde{w}(x, t)=\int_{0}^{1}\{\theta(x-\xi, t)+\theta(x+\xi, t)\} d \xi,
$$

we can easily obtain

$$
\tilde{w}(x, t) \equiv 1 \text {. }
$$

By our early analysis we know that $v(x, t)$, being the limit of $\left\{v^{(n)}(x, t)\right\}$, satisfies (2.1)-(2.4) with all superscripts removed. An argument similar to that for obtaining (2.13) leads to

$$
\|\phi\| \leq \frac{\|r\|\|g\|+2(\|\beta\|+\|\gamma\|)(\|f\|+C \sqrt{t}\|r\|\|F\|)}{1-4 C \sqrt{t}(\|\beta\|+\|\gamma\|)},
$$

for $4 C \sqrt{t}(\|\beta\|+\|\gamma\|)<1$. Under assumption (A1), using the representation of $v(x, t)$

$$
\begin{aligned}
v(x, t)= & w(x, t)-2 \int_{0}^{t} \theta(x, t-\tau) \phi_{1}(\tau) d \tau \\
& +2 \int_{0}^{t} \theta(x-1, t-\tau) \phi_{2}(\tau) d \tau+z(x, t),
\end{aligned}
$$

with (2.2), (2.35), and (2.36), we obtain $v(x, t) \geq f_{\min }-\frac{4 C \sqrt{t}[\|r\|\|g\|+2(\|\beta\|+\|\gamma\|)(\|f\|+C \sqrt{t}\|r\|\|F\|)]}{1-4 C \sqrt{t}(\|\beta\|+\|\gamma\|)}+z(x, t)$,

where

$$
f_{\min }=\min _{x \in[0,1]}\{f(x)\}
$$

Assumption (A2). We assume that $F(x, t) \geq 0$ in $[0,1] \times[0, T]$, and

$$
f_{\min }>\frac{4 C \sqrt{T}[\|r\|\|g\|+2(\|\beta\|+\|\gamma\|)(\|f\|+C \sqrt{T}\|r\|\|F\|)]}{1-4 C \sqrt{\varepsilon}(\|\beta\|+\|\gamma\|)} .
$$


ThEOREM 2. Under assumptions (A1) and (A2), there exists a unique solution pair $(u, p)$ of $(1.1)-(1.5)$ in $[0,1] \times[0, \varepsilon]$ for the mixed boundary value case.

Proof. Under these two assumptions, it follows from (1.15)-(1.19), (2.22) and (2.38) that

$$
v(x, t)>0 \text { in }[0,1] \times[0, \varepsilon] \text {, }
$$

and thus

$$
r(t)=[E(t)]^{-1} \int_{0}^{s(t)} v(x, t) d x>0,
$$

we can use (1.13), (1.14) to find $(u, p)$.

For the important special case $\alpha_{1}=\alpha_{2}=1, \beta_{1}=\beta_{2}=\gamma_{1}=\gamma_{2}=0$, i.e., the second boundary value problem, the positivity of $r(t)$ can be obtained by assuming, in addition to assumption (A1), that $g_{1} \leq 0, g_{2} \geq 0, F \geq 0$, $f \geq 0$, but not all identically zero.

REMARK. Using the existing results on the system described by (1.8)-(1.11), a sequence of solution pairs $\left\{\left(r^{n}, v^{n}\right)\right\}$ can be generated. It can be shown that the limit of this sequence also satisfies (1.12). The existence, uniqueness, etc., then can be addressed in terms of a weak solution. This provides a new proof to Theorem 1. The merit of the classical approach we took here is that the positivity of $v$ can be obtained together with other properties. This has enabled us to derive a more concise proof for the second boundary value case than through the weak solution.

\subsection{Continuous dependence of $(u, p)$ upon the data}

TheOREм 3. The solution pair $(u, p)$ obtained in Section 2.1 depends continuously upon the data.

Proof. Let

$$
\left(f, F, g_{1}, g_{2}, \beta_{1}, \beta_{2}, \gamma_{1}, \gamma_{2}, E, s\right)
$$

and

$$
\left(\bar{f}, \bar{F}, \bar{g}_{1}, \bar{g}_{2}, \bar{\beta}_{1}, \bar{\beta}_{2}, \bar{\gamma}_{1}, \bar{\gamma}_{2}, \bar{E}, \bar{s}\right)
$$

be two sets of data. In what follows we shall use $\psi$ and $\bar{\psi}$ to denote quantities associated with these two sets of data, respectively. Also, we shall use $M_{i}, N_{i}, i=1,2, \ldots$, to denote some positive constants.

It is easy to see that if $\left\|\bar{\psi}_{2}\right\| \leq M,\left\|\psi_{1}\right\| \leq N$, then

$$
\left\|\psi_{1} \psi_{2}-\bar{\psi}_{1} \bar{\psi}_{2}\right\| \leq M\left\|\psi_{1}-\bar{\psi}_{1}\right\|+N\left\|\psi_{2}-\bar{\psi}_{2}\right\| \text {. }
$$

From (2.2) we see, similar to (2.8), that

$$
\|w-\bar{w}\| \leq 2\|f-\bar{f}\| .
$$


It follows from (2.3) and (2.42) that

$$
\|z-\bar{z}\| \leq M_{1}\|r-\bar{r}\|+N_{1}\|F-\bar{F}\| .
$$

Applying (2.42), (2.43) and (2.44) to (2.4), we obtain

$$
\begin{aligned}
\|\phi-\bar{\phi}\| \leq & M_{2}\|f-\bar{f}\|+M_{3}\|g-\bar{g}\|+M_{4}\|F-\bar{F}\|+M_{5}\|\beta-\bar{\beta}\| \\
& +M_{6}\|\gamma-\bar{\gamma}\|+M_{6}\|r-\bar{r}\|+M_{8}\|\phi-\bar{\phi}\|,
\end{aligned}
$$

where

$$
M_{8}=4 C \sqrt{t}(\|\beta\|+\|\gamma\|),
$$

and hence

$\|\phi-\bar{\phi}\| \leq\left(1-M_{8}\right)^{-1} M_{9}(\|f-\bar{f}\|+\|g-\bar{g}\|+\|F-\bar{F}\|+\|\beta-\bar{\beta}\|+\|\gamma-\bar{\gamma}\|+\|r-\bar{r}\|)$,

for $M_{8}<1$. It follows from (1.12) and (2.42) that

$$
\|r-\bar{r}\| \leq M_{10}\|E-\bar{E}\|+M_{11} \int_{0}^{t}\|v-\bar{v}\| d x+M_{12} \int_{s(t)}^{\bar{s}(t)}\|\bar{v}\| d x .
$$

Using (2.42)-(2.47) with the representations of $v$ and $\bar{v}$, we can derive $\|r-\bar{r}\| \leq M_{13}(\|f-\bar{f}\|+\|g-\bar{g}\|+\|F-\bar{F}\|+\|\beta-\bar{\beta}\|+\|\gamma-\bar{\gamma}\|+\|E-\bar{E}\|+\|s-\bar{s}\|)$,

and this implies that $r(t)$ depends continuously upon the data. Then we see from (2.37), (2.43)-(2.46) that $v(x, t)$ depends continuously upon the data.

Differentiating (2.34) with respect to $x$, we can obtain a representation for $v_{x}(x, t)$, and then $v_{x}(s(t), t)$. Using an argument similar to what we demonstrated above, we can prove that $v_{x}$ depends continuously upon the data as well. Then the continuous dependence of $r^{\prime}(t)$ upon the data follows from (1.12) and (2.29). The continuous dependence of $v, r$, and $r^{\prime}$ upon the data guarantees the continuous dependence of $(u, p)$ upon the data.

\subsection{Regularity of the solution pair $(u, p)$}

THEOREM 4. If $f, F, g_{1}, g_{2}, \beta_{1}, \beta_{2}, \gamma_{1}, \gamma_{2}, E, s \in C^{\infty}$ then $u, p \in C^{\infty}$.

Proof. We have proved by $(2.29)$ that $r(t) \in C^{1}[0, \varepsilon]$. Differentiating (1.8) with respect to $t$, and letting $U=v_{t}$, we obtain

$$
\begin{gathered}
U_{t}=U_{x x}+(r F)_{t}, \quad 0<x<1, \quad 0<t \leq T, \\
U(x, 0)=f^{\prime \prime}(x)+r(0) F(x, 0), \quad 0 \leq x \leq 1, \\
U_{x}(0, t)+\beta_{1}(t) U(0, t)+\gamma_{1}(t) U(1, t)=\left(r g_{1}\right)^{\prime}-\beta_{1}^{\prime} v(0,1)-\gamma_{1}^{\prime} v(1, t), \\
0<t \leq T,
\end{gathered}
$$




$$
\begin{gathered}
U_{x}(1, t)+\beta_{2}(t) U(0, t)+\gamma_{2}(t) U(1, t)=\left(r g_{2}\right)^{\prime}-\beta_{2}^{\prime} v(0,1)-\gamma_{2}^{\prime} v(1, t) \\
0<t \leq T \\
r^{\prime}(t)=[E(t)]^{-2}\left[E(t)-E^{\prime}(t)\right] \int_{0}^{s(t)} U d x \\
+[E(t)]^{-1} U(s(t), t) s^{\prime}(t), \quad 0<t \leq T, 0<s(t) \leq 1
\end{gathered}
$$

This equation with all its boundary conditions are analogous to (1.8)(1.12). Under the same assumptions and using the same techniques for acquiring (2.29), we can show that $r^{\prime}(t) \in C^{1}[0, \varepsilon]$, i.e., $r(t) \in C^{2}[0, \varepsilon]$. Upon repetition of this procedure, we can prove that $r(t) \in C^{n}[0, \varepsilon]$, $n=1,2, \ldots$. The same argument applies to $v(x, t)$ as well. Thus, the regularity of $(u, p)$ follows from (1.13) and (1.14).

\subsection{Extension of the local solution of the global solution}

The solution pair $(v, r)$ obtained in the previous sections is a local solution in $[0,1] \times[0, \varepsilon]$. It can be extended to $[0,1] \times[0, T]$ for any $T>0$ in the following way. Defining $W(x, t)=v(x, t+\varepsilon)$, for $\alpha_{1}(t)=\alpha_{2}(t)=1$, we obtain from (1.8)-(1.12)

$$
\begin{gathered}
W_{t}=W_{x x}+r(t+\varepsilon) F(x, t+\varepsilon), \quad 0<x<1,0<t \leq T-\varepsilon, \\
W(x, 0)=v(x, \varepsilon), \quad 0 \leq x \leq 1, \\
\begin{aligned}
& W_{x}(0, t)+\beta_{1}(t+\varepsilon) W(0, t)+\gamma_{1}(t+\varepsilon) W(1, t)= r(t+\varepsilon) g_{1}(t+\varepsilon), \\
& 0<t \leq T-\varepsilon, \\
& W_{x}(1, t)+\beta_{2}(t+\varepsilon) W(0, t)+\gamma_{2}(t+\varepsilon) W(1, t)= r(t+\varepsilon) g_{2}(t+\varepsilon), \\
& 0<t \leq T-\varepsilon, \\
& r(t+\varepsilon)=[E(t+\varepsilon)]^{-1} \int_{0}^{s(t+\varepsilon)} W d x, \\
& 0<t \leq T-\varepsilon, 0<s(t+\varepsilon) \leq 1 .
\end{aligned}
\end{gathered}
$$

Recalling that, under the assumptions (A1) and (A2), the $\varepsilon$ obtained depends on the global behaviour of the data, we see from the proof given above that $\exists \varepsilon>0$ such that $(2.54)-(2.58)$ has a unique solution pair $(W, r(t+\varepsilon))$ in $[0,1] \times[0, \varepsilon]$, i.e., $(1.8)-(1.12)$ has a unique solution in $[0,1] \times[0,2 \varepsilon]$. Repeating the same procedure a finite number of times, the solution can be extended to $[0,1] \times[0, T]$ for any $T>0$. Hence, the solution pair exists on $[0,1] \times[0, T]$. 


\section{The first boundary value case}

When $\alpha_{1}(t)=\alpha_{2}(t) \equiv 0$, and $\beta_{1} \gamma_{2} \neq \beta_{2} \gamma_{1},(1.1)-(1.5)$ can be reduced to the following first boundary value problem.

$$
\begin{array}{rc}
v_{t}=v_{x x}+r(t) F(x, t), & 0<x<1,0<t \leq T, \\
v(x, 0)=f(x), & 0 \leq x \leq 1, \\
v(0, t)=r(t) g_{1}(t), & 0<t \leq T, \\
v(1, t)=r(t) g_{2}(t), & 0<t \leq T, \\
r(t)=[E(t)]^{-1} \int_{0}^{s(t)} v d x, & 0<t \leq T, 0<s(t) \leq 1 .
\end{array}
$$

Assuming the existence of $r(t)$ and following [1], we can express the solution of (3.1)-(3.5) as

$$
\begin{aligned}
v(x, t)= & \int_{0}^{1}\{\theta(x-\xi, t)-\theta(x+\xi, t)\} f(\xi) d \xi \\
& -2 \int_{0}^{t} \frac{\partial \theta}{\partial x}(x, t-\tau) r(\tau) g_{1}(\tau) d \tau \\
& +2 \int_{0}^{t} \frac{\partial \theta}{\partial x}(x-1, t-\tau) r(\tau) g_{2}(\tau) d \tau \\
& +\int_{0}^{t} \int_{0}^{1}\{\theta(x-\xi, t-\tau)-\theta(x+\xi, t-\tau)\} r(\tau) F(\xi, \tau) d \xi d \tau
\end{aligned}
$$

\subsection{Existence and uniqueness}

The problem (3.1)-(3.5) is a special case of (1.8)-(1.12). We now prove Theorem 1 for this case.

Proof. In (1.16)-(1.20), letting $\alpha_{1}=\alpha_{2}=\beta_{2}=\gamma_{1}=0, \beta_{1}=\gamma_{2}=1$, we have a sequence of iterations approximating $(v, r)$. The same procedure used in Section 2.1 yields the proof.

THEOREM 5. If $g_{1}, g_{2}, F, f \geq 0, E(0)=\int_{0}^{s(0)} f(x) d x>0$, and $E(t)>0$, then $v \geq 0, r(t)>0$.

Proof. Since $r^{(0)}=1$, it follows from the iteration procedure that $r^{(n)} \geq$ $0, v^{(n)} \geq 0$, and this implies that $r(t) \geq 0, v \geq 0$. Note that $r(0)=1$. If there exists a $t_{0}>0$ such that $r\left(t_{0}\right)=0$, then it follows from (3.5) that $v\left(x, t_{0}\right) \equiv 0$ and, accordingly, $v_{x}\left(x, t_{0}\right) \equiv 0$. In particular $v_{x}\left(0, t_{0}\right)=0$. 
However, according to the assumption, we see that $v\left(0, t_{0}\right)$ is a minimum of $v(x, t)$. It follows from the Hopf's lemma [1], [13] that $v_{x}\left(0, t_{0}\right)>0$, which is a contradiction, and thus the proof follows.

THEOREM 6. Under the assumption (A1) and that of Theorem 5, there exists a unique solution pair $(u, p)$ of (1.1)-(1.5) for the first boundary value case.

Proof. The proof follows from Theorem 5 and (1.13) and (1.14).

3.2. Continuous dependence of $(u, p)$ upon the data

Theorem 7. The solution pair $(u, p)$ obtained in Section 3.1 depends continuously upon the data.

Proof. The proof is similar to that of Theorem 3.

3.3. Regularity of the solution pair $(u, p)$

THEOREM 8. If $f, F, g_{1}, g_{2}, s, E \in C^{\infty}$, then $u, p \in C^{\infty}$.

Proof. The proof is similar to that of Theorem 4.

3.4. Extension of local solution to global solution

The local solution pair $(u, p)$ can be extended to the global solution for $(x, t) \in[0,1] \times[0, T]$ for any $T>0$ in the same fashion as we demonstrated in Section 2.4.

\section{Acknowledgement}

The authors would like to thank the referees for their careful reading of this paper and constructive suggestions that we have included in the remark following the proof of Theorem 2 .

This research was supported in part by the National Science Foundation Grant DMS-8901301 and the Texas Higher Education Coordinating Board Grant \#003581-006.

\section{References}

[1] J. R. Cannon, The one dimensional heat equation, Encyclopedia of mathematics and its application 23, (Addison-Wesley, Monlo Park, Calif., 1984).

[2] J. R. Cannon, "The solution of the heat equation subject to the specification of energy", Quart. Appl. Math. 21 (1963) 155-160. 
[3] J. R. Cannon and J. van der Hoek, "The classical solution of the one-dimensional twophase Stefan problem with energy specification", Annali di Mat. Pura ed appl., (IV) 130 (1982) 385-398.

[4] J. R. Cannon and J. van der Hoek, "The one phase Stefan problem subject to the specification of energy", J. Math. Anal. and Appl. 86 (1982) 281-291.

[5] J. R. Cannon and J. van der Hoek, "Diffusion subject to the specification of mass", $J$. Math. Anal. Appl. 115 (1986) 517-529.

[6] J. R. Cannon, S. P. Esteva and J. van der Hoek, “A Galerkin procedure for the diffusion equation subject to the specification of mass", SIAM J. Numer. Anal. 24 (1987) 499-515.

[7] J. R. Cannon and Yanping Lin, "Determination of parameter $p(t)$ in some quasi-linear parabolic differential equations", Inverse Problems 4 (1988) 35-45.

[8] J. R. Cannon and Yanping Lin, "Determination of parameter $p(t)$ in Hölder classes for some semilinear parabolic equations", Inverse Problems 4 (1988) 595-606.

[9] J. R. Cannon and D. Zachmann, "Parameter determination in parabolic partial differential equations from overspecified boundary data", Int. J. Eng. Sci. 20 No. 6 (1982) 779-788.

[10] V. Capasso and $K$. Kunisch, "A reaction-diffusion system arising in modeling manenvironment diseases", Quart. Appl. Math. 46 (1988) 431-450.

[11] W. A. Day, "Existence of a property of solutions of the heat equation to linear thermoelasticity and other theories", Quart. Appl. Math. 40 (1982) 319-330.

[12] K. L. Deckert and C. G. Maple, "Solution for diffusion with integral boundary conditions", Proc. lowa Acad. Sci. 70 (1963) 354-361.

[13] A. Friedman, Partial differential equation of parabolic type (Prentice-Hall, Inc., 1964).

[14] N. I. Ionkin, "Solution of the boundary value problem in heat conduction with a nonclassical boundary condition", (English trans.) Diff. Eq. 13 (1977) 204-211.

[15] N. I. Ionkin, "Stability of a problem in heat transfer theory with a non-classical boundary condition", (English trans.) Diff. Eq. 15 (1980) 911-914.

[16] L. A. Kamynin, "A boundary value problem in the theory of heat conduction with a non-classical boundary condition", (English translation) USSR Comp. and Math. Phys. 4 (1964) 33-59.

[17] O. A. Ladyzenskaja, V. A. Solonikov and N. N. Uralceva, "Linear and quasilinear equations of parabolic type", A.M.S. Tran. Math. Mono. 23, Providence, R.I. 1968.

[18] J. L. Lions and E. Magenes, Non-homogeneous boundary valued problems and applications, Vols. I-III (Springer-Verlag, Berlin, 1972).

[19] V. P. Mikhailov, Partial differential equations (MIR Publishers, Moscow, 1979).

[20] A. I. Prilepko and D. G. Orlovskii, "Determination of the evolution parameter of an equation and inverse problems of mathematical physics. I", Diff. Eqs. 21 (1985) 119129.

[21] A. I. Prilepko and D. G. Orlovskii, "Determination of a parameter in an evolution equation and inverse problems of mathematical physics. II", Diff. Eqs. 21 (1985) 694701.

[22] W. Rundell, "Determination of an unknown nonhomogeneous term in a linear partial differential equation from overspecified boundary data", Applicable Analysis 10 (1980) 231-242.

[23] K. L. Teo, Computational methods for optimizing distributed systems (Academic Press, Orlando, 1984). 